\title{
Kleinwinkelbeugung mit Elektronenstrahlen ${ }^{1}$
}

\author{
Von H. Mahl und W. Weitsch \\ Abteilung für Elektronenoptik, Fa. CarL ZEIss, Oberkochen (Württ.) \\ (Z. Naturforschg. 15 a, 1051-1055 [1960]; eingegangen am 9. August 1960)
}

\begin{abstract}
Es wird eine Elektronenbeugungsanordnung hoher Auflösung angegeben, die durch geringe Eingriffe aus dem AEG-ZEISS-Elektronenmikroskop EM 8 entsteht und die es gestattet, KleinwinkelBeugungsreflexe sichtbar zu machen, die Perioden bis über $600 \AA \AA$ entsprechen. Einige Beispiele von KW-Beugungserscheinungen an orientierten und nichtorientierten Aufdampfschichten sowie an organischen Substanzen werden gezeigt. Die Beugungsdiagramme sind auf dem Leuchtschirm direkt beobachtbar und erfordern Belichtungszeiten von nur wenigen Sekunden. Auf einige Verbesserungsmöglichkeiten wird hingewiesen.
\end{abstract}

Während auf dem Gebiet der Strukturforschung mittels Röntgen-Strahlen die Kleinwinkel-Beugungserscheinungen in großem Umfang zur Untersuchung großer Perioden bei organischen Substanzen verwendet werden, sind unseres Wissens Versuche zur Kleinwinkelbeugung mit Elektronenstrahlen noch nicht bekannt geworden. Bisher wurden im allgemeinen Elekronenbeugungserscheinungen untersucht, bei denen Perioden bis nicht viel über $10 \AA$ wirksam sind. Wohl sind Elektronenbeugungsgeräte hoher Auflösung ${ }^{2}$ bekannt geworden; sie wurden jedoch nicht zur Untersuchung der Beugungserscheinungen in unmittelbarer Nähe des Primärstrahls angewandt. sondern zur Auflösung der Feinstruktur von Weitwinkelreflexen oder zur genauen Bestimmung von Gitterkonstanten-Differenzen.

Die Forderungen, die man an ein Elektronenbeugungsgerät stellen muß, um in das Gebiet großer Perioden bis etwa $1000 \AA$ vordringen zu können, ergeben sich aus der BraGgschen Gleichung

$$
2 d \sin \vartheta / 2=\lambda .
$$

Für $d=1000 \AA$ und $\lambda=0,06 \AA$ (entsprechend $40 \mathrm{kV}$ Elektronengeschwindigkeit) wird der Beugungswinkel $\vartheta=6 \cdot 10^{-5} \quad(=12$ Bogensekunden $)$. Bei einer effektiven Kameralänge von z. B. $5 \mathrm{~m}$ ergibt sich daraus ein Reflexabstand von $0,3 \mathrm{~mm}$ von der Primärstrahlachse. Damit dieser Reflex beobachtet werden kann, muß der Primärfleckradius kleiner sein als dieser Wert, die Auflösungszahl also kleiner als $6 \cdot 10^{-5}$.

1 H. Mahl u. W. Weitsch, Naturwiss. 47, 301 [1960] (kurze Originalmitteilung).

2 Als Auflösungszahl $n_{\mathrm{A}}$ bezeichnet man (BAuer ${ }^{3}$ ) das Verhältnis von Primärfleckradius $r_{\mathrm{R}}$ zu effektiver Kameralänge $L$; für die günstigste bis 1957 bekanntgewordene Anordnung hat sie den Wert $3 \cdot 10^{-6}$ (TanaKa und HashIмото $\left.{ }^{4}\right)$.
Diese Forderungen lassen sich ohne große Schwierigkeit erfüllen. Die große effektive Kameralänge kann durch entsprechende Vergrößerung des Beugungsbildes erreicht werden, der feine Primärstrahl durch Verwendung einer genügend feinen Elektronenstrahlquelle, die sich durch elektronenoptische Verkleinerung des engsten Strahlquerschnittes der Glühkathode verwirklichen läßt.

\section{Versuchsanordnung}

In Abb. 1 ist schematisch der für unsere Versuche benutzte Strahlengang mit den wesentlichen geometrischen und elektronenoptischen Daten wiedergegeben, auf die sich auch die im folgenden benutzten Buchstaben beziehen. Er läßt sich durch verhältnismäßig geringe Eingriffe im AEG-ZEISS-Elektronenmikroskop EM 8 verwirklichen. Das Objekt befindet sich zwischen Beugungslinse und Projektiv in einem verschiebbaren Halter, der normalerweise zur Aufnahme der Bereichsblende bestimmt ist. Der im normalen Abbildungsstrahlengang zum Einschwenken der Bereichsblende dienende Mechanismus wurde so umgebaut, $\mathrm{da} ß$ man oberhalb des $\mathrm{Ob}$ jekts eine Vorblende von $50 \mu$ einschwenken kann. Als Strahlquelle dient der Brennfleck des Mikroskopobjektivs. Da die Anodenblende von $80 \mu$ nur wenig mehr als zur Hälfte mit hoher Intensität ausgeleuchtet wird, ergibt sich für diesen Brennfleck ein Durchmesser von etwa $d_{0}=60 \mu f_{\mathrm{Obj}} / L_{1}=3,4 \mu$, eine Größe, die sich im BoErschschen Beugungsbild durch Vergleich mit dem Durchmesser der Kontrastblende bestätigen läßt. In einem Strahlengang, wie er erstmalig von LeBedefF ${ }^{5}$ angegeben wurde, bildet die Beugungslinse diesen Brennfleck etwa im Maßstab $\left(L_{3}+L_{4}+L_{5}\right) / L_{2}=2,3: 1$ in die konjugierte Ebene des langbrennweitigen Projektivs ab.

3 E. Bauer, Elektronenbeugung, Verlag Moderne Industrie, München 1958, S. 114.

${ }^{4}$ K. Tanaka u. H. Hashimoto, Rev. Sci. Instrum. 24, 669 [1953].

5 A. A. Lebedeff, Nature, Lond. 128, 491 [1931]. 


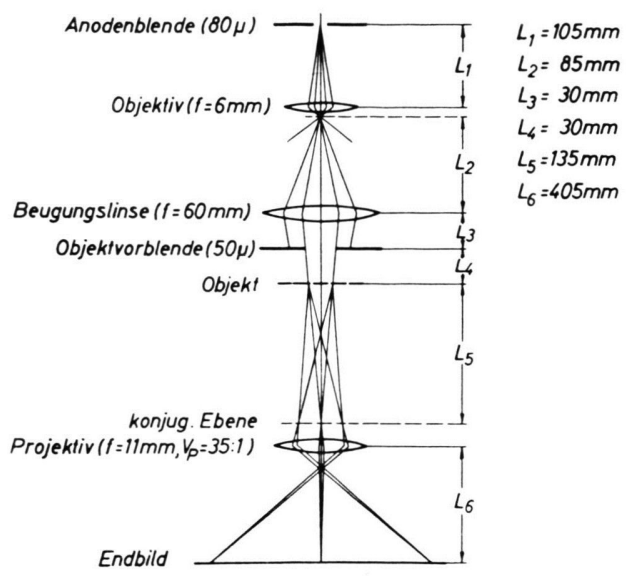

Abb. 1. Strahlengang für Elektronen-Kleinwinkelbeugung.

Das in dieser Ebene entstehende, sehr kleine Beugungsdiagramm wird dann auf den Leuchtschirm bzw. das Photomaterial 35-fach vergrößert abgebildet. Mit diesem Strahlengang berechnet sich ein Primärfleckdurchmesser $d_{\mathrm{R}}=d_{0} \cdot 2,3 \cdot 35=280 \mu$, ein Wert, der mit der Beobachtung ohne Objekt übereinstimmt. Die effektive Kameralänge ist $L=V_{\mathrm{P}} L_{5}=4,7 \mathrm{~m}$. Die oben definierte Auflösungszahl $n_{\mathrm{A}}=r_{\mathrm{R}} / L$ beträgt daher $3 \cdot 10^{-5}$. Somit müßten sich noch Reflexe von Perioden weit über $1000 \AA$ beobachten lassen. Einbringen eines Objekts in den Strahlengang bewirkt jedoch eine je nach der Art des Objekts mehr oder weniger große Verbreiterung des Primärflecks. Unter Berücksichtigung dieser Verbreiterung ergibt sich, daß eine Auflösung der Reflexe von Perioden bis etwa $800 \AA$ A möglich ist. Zum Vergleich sei erwähnt, daß im üblichen Boerschschen Beugungsbild des AEG-ZEISS-Elektronenmikroskops bei einer effektiven Kameralänge von 0,5 m Ringe beobachtet werden können, die einer Periode von ca. $80 \AA \AA$ entsprechen.

Die beschriebene Anordnung bietet überdies die Möglichkeit einer Beobachtung des Objekts bei geringer elektronenoptischer Vergrößerung. Schaltet man nämlich die Beugungslinse $a b$, so erzeugt der Objektivbrennfleck in der Gegenstandsebene des langbrennweitigen Projektivs ein Schattenbild des Objekts, das dann durch das Projektiv auf den Leuchtschirm vergrößert abgebildet wird. Die Gesamtvergrößerung dieses Schattenbildes beträgt etwa $70: 1$. Schaltet man statt dessen das kurzbrennweitige Projektiv ein, so ergibt sich eine Vergrößerung von etwa $200: 1$.

Mit der geschilderten Anordnung konnten an verschiedenen anorganischen und organischen Objekten Kleinwinkel-Beugungserscheinungen sichtbar gemacht werden ${ }^{1}$, wobei das 20 -fach vergrößernde Einblickmikroskop des Elektronenmikroskops die direkte Beobachtung wesentlich erleichterte oder zum Teil auch erst ermöglichte.

* Abb. 2 und 4-11 auf Tafel S. 1054 a, b.

${ }^{6}$ Zur deutlichen Positiv-Wiedergabe der KW-Diagramme war es wegen des stark ansteigenden Untergrundes in Primärflecknähe erforderlich, bei der Vergrößerung der Nega-

\section{Ergebnisse}

\section{a) Anorganische Aufdampfschichten}

Zunächst wurden KW-Beugungserscheinungen an einer Anzahl von Aufdampfschichten untersucht. Die beobachteten Beugungserscheinungen sind aber nur bei solchen Schichten klar und übersichtlich, die auf amorpher Unterlage erzeugt wurden und aus isoliert liegenden, statistisch verteilten Körnchen bestehen (Abb. $2 \mathrm{a}^{*}$ ). An solchen Schichten treten immer 1 oder 2 mehr oder weniger diffuse Ringe auf, wie Abb. 2 b an einer Thalliumchloridschicht (auf Kohlefolie aufgedampft) zeigt ${ }^{6}$. Nach der Braggschen Formel entsprechen den beiden Ringen der Thalliumchloridschicht mittlere Periodenlängen von 600 bzw. $250 \AA$. Diese Werte stimmen mit den mittleren Kornabständen, aber auch mit den Korngrößen, einigermaßen überein. Die von diesem Präparat ermittelte Größenverteilungskurve (es wurden über 2000 Teilchen ausgezählt) ist in Abb. 3 wiedergegeben. Die Verteilungskurve zeigt 2 Maxima, die bei ca. 520 und $200 \AA$ A liegen. Auf Formvar aufgedampfte Goldschichten, die wesentlich feinkörniger sind als die Thalliumchloridschicht, zeigen jeweils einen diffusen Ring (z. B. Abb. 4 b), aus dem eine mittlere Periodenlänge folgt, die bei mehreren sonst ähnlichen Schichten zwischen 80 und $120 \AA$ liegt; dies stimmt ebenfalls etwa mit den Kornabständen und Korngrößen (vgl. Abb. 4 a) überein.

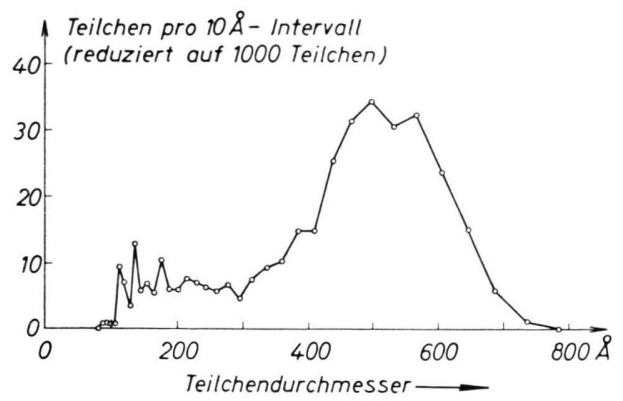

Abb. 3. Größenverteilung der Aufdampfkörner in Abb. 2 a.

Diese und andere Ergebnisse an Aufdampfschichten auf amorpher Unterlage sind im oberen Teil der Tab. 1 zusammengestellt. Dabei zeigt sich, daß die aus den KW-Beugungsdiagrammen Abb. 5 a, 5 b, 5 c

tive die Belichtungszeit im Zentrum gegenüber den äußeren Bereichen zu verlängern; dies konnte durch eine Irisblende erreicht werden, die während des Belichtungsvorganges allmählich zugezogen wurde. 


\begin{tabular}{|c|c|c|c|c|c|}
\hline \multirow{2}{*}{ Präparat } & \multicolumn{2}{|c|}{ KW-Diagramme } & \multicolumn{3}{|c|}{ EM-Bild } \\
\hline & Typ & mittl. Periodenlänge & Typ & Korngrößen & Kornabstände \\
\hline TlCl auf Kohlefolie & 2 Ringe & $600,250 \AA$ & $\begin{array}{l}\text { einzeln liegende } \\
\text { Körner }\end{array}$ & $\begin{array}{l}\quad 70-750 \AA \\
\text { Häufigkeitsmaxima } \\
\text { bei } 520 \text { und } 200 \AA\end{array}$ & $300-1000 \AA ̊$ \\
\hline $\begin{array}{l}\text { Au auf Formvar } \\
\text { oder Kohlefolie }\end{array}$ & 1 Ring & $80-120 \AA \AA$ & ” & $50-120 \AA$ & $50-200 \AA$ \\
\hline AgJ auf Formvar & 1 Ring & $650 \AA \AA$ & $"$ & $70-600 \AA$ & $200-800 \AA$ \\
\hline $\mathrm{Ag}_{2} \mathrm{~S}$ auf Formvar & $\begin{array}{l}1 \text { Ring } \\
\text { sehr breit }\end{array}$ & $260 \AA$ & $"$ & $100-220 \AA$ & $150-350 \AA$ \\
\hline $\mathrm{Pb}$ auf Formvar & 2 Ringe & $500,250 \AA$ & ” & $100-300 \AA$ & $200-600 \AA$ \\
\hline $\begin{array}{cl}\mathrm{Au} \text { auf } & \text { grobkörnig } \\
\mathrm{NaCl} & \text { feinkörnig }\end{array}$ & $\begin{array}{c}\text { Textur } \\
,\end{array}$ & $\begin{array}{c}160,200 \AA \AA \\
35,100-130 \AA\end{array}$ & " & $\begin{array}{l}40-180 \AA \\
20-80 \AA\end{array}$ & $\begin{array}{l}80-300 \AA \\
50-150 \AA\end{array}$ \\
\hline $\mathrm{Pd}$ auf $\mathrm{NaCl}$ & $"$ & $120,200 \AA$ & $\begin{array}{l}\text { zusammenhängende } \\
\text { körnige Schicht }\end{array}$ & - & - \\
\hline
\end{tabular}

Tab. 1. Zusammenstellung der aus KW-Diagrammen und EM-Aufnahmen ermittelten mittleren Periodenlängen bzw. Korngrößen und -abstände (kleinste und größte Werte) aller untersuchten Aufdampfschichten.

erhaltenen Werte etwas besser mit den Kornabständen übereinzustimmen scheinen als mit den Korngrößen. Statistische Untersuchungen wurden aber, bis auf die erwähnte, in den anderen Fällen nicht durchgeführt. Von dem Originalnegativ der Abb. 5 c wurde eine Mikrophotometer-Registrierkurve aufgenommen, die in Abb. 6 wiedergegeben ist.

Wesentlich kompliziertere KW-Diagramme zeigen Aufdampfschichten, die auf einer kristallinen Unterlage, z. B. auf einer NaCl-Spaltfläche, erzeugt wurden ${ }^{7}$. Hier scheint, wie die KW-Diagramme zeigen, im allgemeinen - wenigstens bereichsweise - eine gewisse Ordnung in der Aufdampfschicht vorzuliegen, selbst wenn dies im normalen Weitwinkeldiagramm oder im elektronenmikroskopischen Bild nicht in Erscheinung tritt. So zeigt eine gröbere Goldschicht (Abb. 7 a), die durch Senkrecht-Bedampfen einer NaCl-Spaltfläche erzeugt wurde, einen Ring (Abb. 7 b), dessen Durchmesser einer mittleren Periode von etwa $160 \AA$ entspricht. An einigen Stellen derselben Schicht zeigt jedoch der Ring eine ungleichmäßige Intensitätsverteilung (Abb. 7 c), wobei die intensivsten Stellen nicht diametral zueinander liegen. (Diese Unsymmetrie ließ sich trotz sorgfältiger Ausleuchtung nicht vermeiden; Abschattung durch Stege des Trägernetzes als Ursache der Unsymmetrie konnte dadurch ausgeschlossen werden, daß das Netz mit dem Objekt nach unten eingebracht wurde.) Auffallend ist, daß bei dieser Schicht in streifenför-

7 Die Schichten wurden mittels eines nachträglich aufgedampften Kohlefilms abgenommen. migen Bereichen eine wesentlich gröbere Granulierung vorliegt (vgl. Abb. 7 a). In einem Fall trat das in Abb. $7 \mathrm{~d}$ wiedergegebene Diagramm auf, in dem die 1., 2. und 3. Ordnung einer Texturperiode von etwa $200 \AA$ zu erkennen ist.

Bei wesentlich feinkörnigeren Schichten, die z. Tl. eine zeilenartige Anlagerung der Goldpartikeln längs der Kanten von Kristallstufen des $\mathrm{NaCl}$ erkennen lassen (Abb. 8 a), wurden außer Beugungsfiguren ähnlich Abb. $7 \mathrm{~b}$ und $7 \mathrm{c}$ (mittlere Periode jedoch nur etwa $120 \AA$ ) noch andersartige Diagramme beobachtet: leicht elliptischer Ring (Abb. 8 b), scharfer radialer Reflex (Abb. $8 \mathrm{c}$ ) sowie Meridian-Reflexe (Abb. $8 \mathrm{~d}$ ). Soweit dabei mittlere Reflexabstände vom Primärfleck angebbar sind, lassen sich bei den feinkörnigen Goldschichten mittlere Perioden von $100-130 \AA$ berechnen. In zwei Fällen wurde im Originalnegativ noch ein sehr schwacher, verhältnismäßig scharfer Ring entsprechend etwa $35 \AA$ festgestellt, der jedoch im Positivbild nicht wiedergegeben werden konnte. Die Angaben über Größen und Abstände der Teilchen in Tab. 1 lassen erkennen, daß auch bei diesen Schichten die Übereinstimmung der berechneten Perioden mit den Kornabständen besser ist als mit den Korngrößen.

Das Weitwinkeldiagramm der hier untersuchten Goldschichten läßt im übrigen in senkrechter Durchstrahlung keine Anzeichen einer Textur erkennen.

Teils ähnliche, teils andersartige Erscheinungen wurden an Palladiumschichten beobachtet, die ebenfalls durch Aufdampfen auf Steinsalzspaltflächen un- 
ter verschiedenen Bedingungen erhalten wurden. In den elektronenmikroskopischen Bildern dieser Schichten läßt sich im allgemeinen nur eine körnige Struktur feststellen, ohne daß man daraus feinere Einzelheiten über die Anlagerung der Partikeln erkennen könnte (Abb.9a). Nur gelegentlich wurde bei Schichten, die auf heißer NaCl-Oberfläche aufgedampft wurden, stellenweise eine Streifenstruktur beobachtet (Abb. 9 b), die vermutlich durch Anlagerung der Kriställchen längs feiner Kristallstufenkanten hervorgerufen wurde.

Das normale Weitwinkeldiagramm zeigt demgegenüber bereits in senkrechter Durchstrahlung, $\mathrm{da} \beta$ selbst bei Pd-Schichten, die auf kalter NaClOberfläche aufgedampft wurden, häufig schon eine deutliche Textur vorliegt (Abb.9c), die auf einen orientierten Kornanteil in der Schicht schließen läßt. Bei Schichten, die oberhalb $250{ }^{\circ} \mathrm{C}$ aufgedampft wurden, tritt immer die bekannte starke Orientierung (Epitaxie) der Aufdampfschicht zur Kristallstruktur der NaCl-Unterlage ein (Abb. 9 d), die im elektronenmikroskopischen Bild aber im allgemeinen nicht in Erscheinung tritt.

Im KW-Diagramm weisen ebenfalls bereits die bei Zimmertemperatur aufgedampften Schichten eine sehr deutliche Textur auf (Abb.9e, 9f). Abb. 9 e zeigt eine überraschende Ähnlichkeit mit sogenannten Meridian-Reflexen in Faserdiagrammen, wie man sie aus der Röntgen-Kleinwinkelbeugung an Hochpolymeren kennt. Der in Abb. 9 f zusätzlich auftretende radiale Reflex kann, wie aus einer Anzahl ähnlicher Aufnahmen hervorging, mit der Symmetrielinie des „Faserdiagramms" von Fall zu Fall verschiedene Winkel einschließen. Es scheint sich hier um zwei voneinander unabhängige Orientierungseffekte zu handeln. Die aus den Abb. 9 e und $9 \mathrm{f}$ berechnete „Faserperiode“ beträgt etwa $120 \AA$.

Das KW-Diagramm Abb. 9 g der erwähnten (bei $200{ }^{\circ} \mathrm{C}$ unter $45^{\circ}$ schräg aufgedampften) Schicht mit stellenweiser Streifenstruktur (Abb. 9 b) zeigt einen ähnlichen radialen Reflex, der wieder verschiedene Winkellagen gegenüber dem „Faserdiagramm“ einnehmen kann. Die Meridian-Reflexe haben hier die Gestalt von Ellipsenbogen angenommen. Aus ihrem diametralen Abstand folgt, in annähernder Übereinstimmung mit dem Streifenabstand in Abb. 9 b, eine Periode von ca. $200 \AA$.

Die bei $250{ }^{\circ} \mathrm{C}$ bzw. $300{ }^{\circ} \mathrm{C}$ aufgedampften Schichten weisen zwei deutlich verschiedene Formen des KW-Diagramms auf. In Abb. $9 \mathrm{~h}$ (schräg unter $45^{\circ}$ aufgedampfte Schicht wie bei Abb. 9 g) haben sich die Meridianreflexe anscheinend zu einer Ellipse zusammengebogen, zu der der Radialreflex wieder verschieden orientiert sein kann. In Abb. 9 i (senkrecht aufgedampfte Schicht) tritt neben einem kreissymmetrischen Streuhof ein besonders scharfer radialer Reflex auf. Dieser starke strichförmige Reflex weist in die Richtung des starken 200-Reflexes, was durch eine Aufnahme derselben Objektstelle bei geringerer Vergrößerung des Beugungsdiagramms (Abb.9 j) nachgewiesen wurde. Es liegt daher offensichtlich ein Kornanteil vor, der parallel zur Würfelkante der $\mathrm{NaCl}-$ Unterlage geordnet ist. Eine Deutung der anderen hier wiedergegebenen KW-Texturdiagramme steht noch aus.

\section{b) Organische Substanzen}

Da die Röntgen-KW-Beugung vor allem für die Untersuchung von aus sehr großen organischen Molekülen bestehenden Substanzen mit großem Erfolg verwendet wird, lag es nahe, entsprechende Versuche auch mit der Elektronen-KW-Beugung durchzuführen. Es zeigt sich aber, daß bei solchen Stoffen nicht ohne weiteres KW-Diagramme erhalten werden können, da hierbei durch die Elektronenbestrahlung sehr starke Aufladungen auftreten, so daß die Scharfstellung des Primärflecks praktisch unmöglich wird. Außerdem scheinen fluktuierende Ladungen in der isolierenden Schicht zu stören, die wechselnde Strahlablenkungen in der Größenordnung der gesuchten Beugungswinkel hervorrufen (MAHL und $\mathrm{W}_{\mathrm{EITSCH}}{ }^{8}$ ). Diese Störeffekte lassen sich jedoch weitgehend beseitigen, wenn die organischen Präparate mit einer dünnen, leitenden Metall- oder Kohlenstoffschicht bedampft werden.

In Abb. 10 sind das elektronenmikroskopische Bild und das KW-Diagramm einer schräg mit Platin bedampften Perlonschicht wiedergegeben ${ }^{9}$. Die eine Symmetrielinie des KW-Diagramms stimmt vermutlich mit der Bedampfungsrichtung überein, da die zu dieser senkrecht liegenden Fibrillen in ihrer Streuwirkung verstärkt werden.

Als weiteres Beispiel zeigt Abb. 11 zwei KW-Diagramme von Kollagenfasern, die ebenfalls schräg mit Platin bedampft waren. Auch hier werden offen-

8 H. Mahl u. W. WeItsch, Optik 17, 107 [1960].

9 Der Perlonfilm wurde dadurch erzeugt, daß eine Lösung von Perlon in Ameisensäure entweder auf einen Glasobjektträger aufgestrichen oder auf Wasser aufgetropft wurde. 


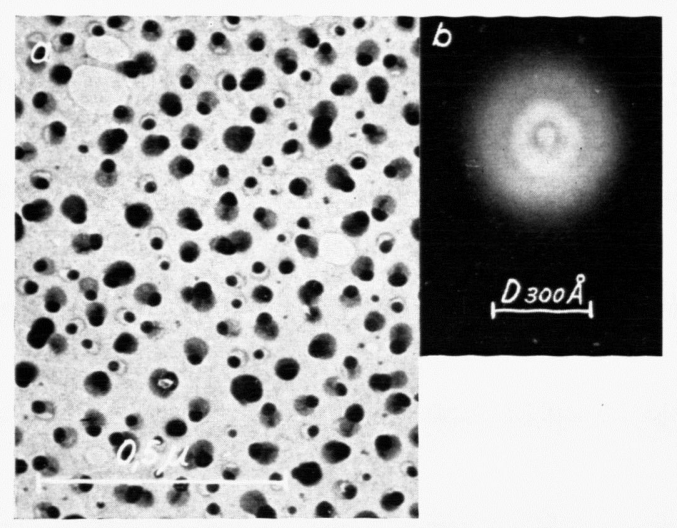

Abb. 2. TlCl auf Kohle aufgedampft. a) EM-Bild, Vergr. $50000: 1$; b) KW-Diagramm, $d D=3000$ (mm Â).

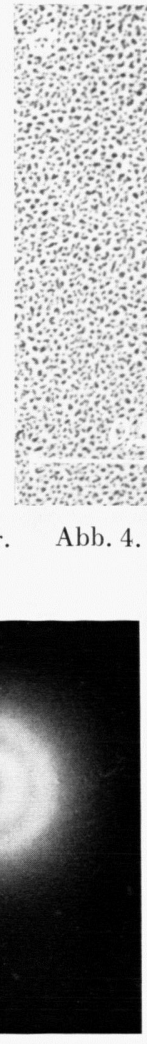

Abb. 5. KW-Diagramme von Aufdampfschichten auf Formvar.

a) $\mathrm{AgJ}$, b) $\mathrm{Ag}_{2} \mathrm{~S}$, c) $\mathrm{Pb}(d D=3000)$.

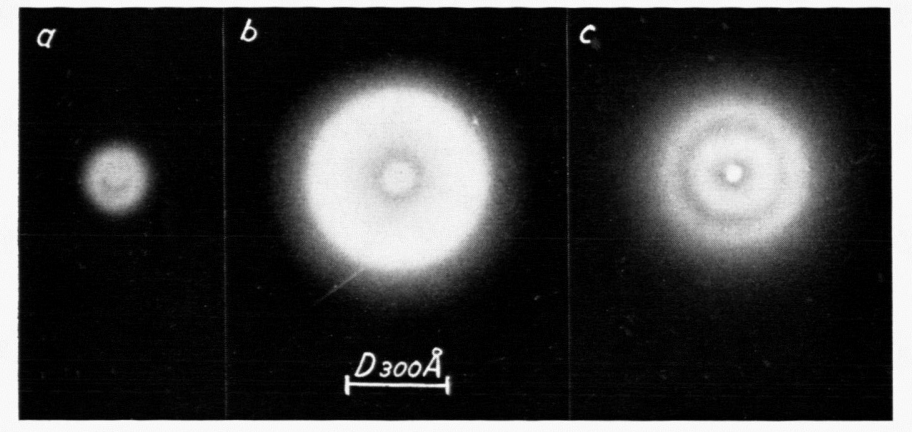

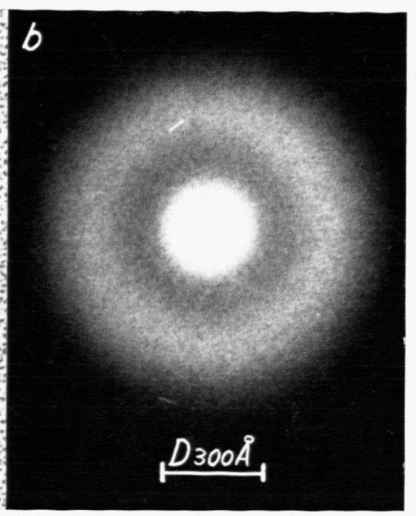

Au auf Formvar aufgedampft. a) EM-Bild, Vergr. $50000: 1$; b) KW-Diagramm, $d D=3000$.

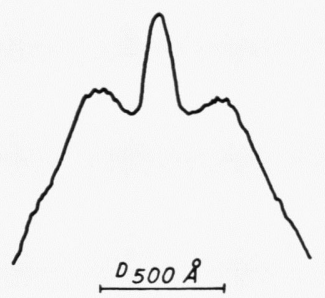

Abb. 6. Mikrophotometerkurve des Originalnegativs von Abb. 5 c.

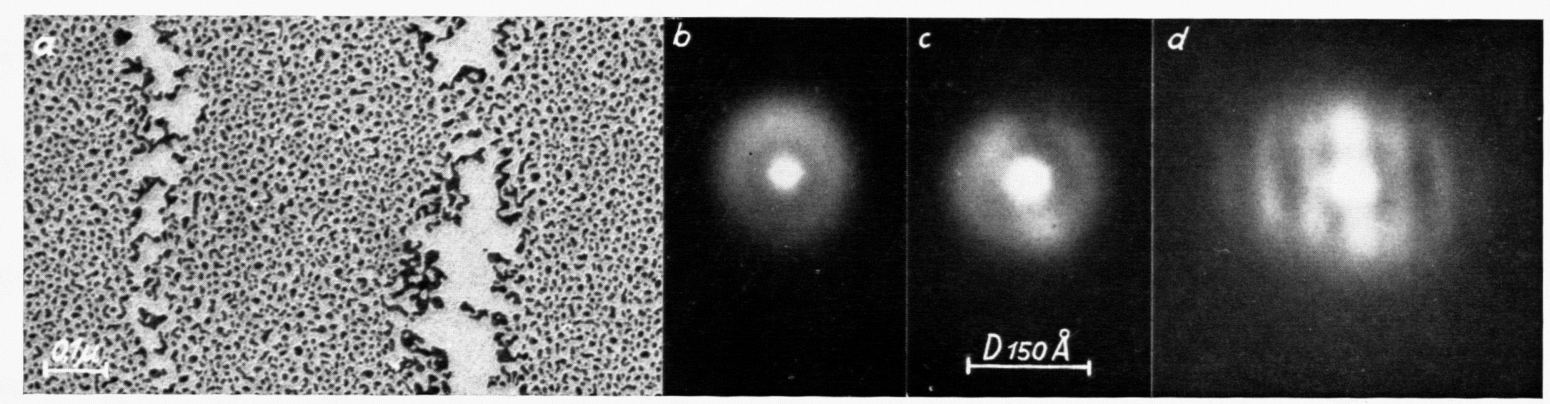

Abb. 7. Au auf NaCl-Spaltfläche bei $20^{\circ} \mathrm{C}$ senkrecht aufgedampft. a) EM-Bild (grobkörnige Schicht), Vergr. 60 000 : 1, $\mathrm{b}-\mathrm{d}) \mathrm{KW}$-Diagramme, $d \mathrm{D}=1800$.

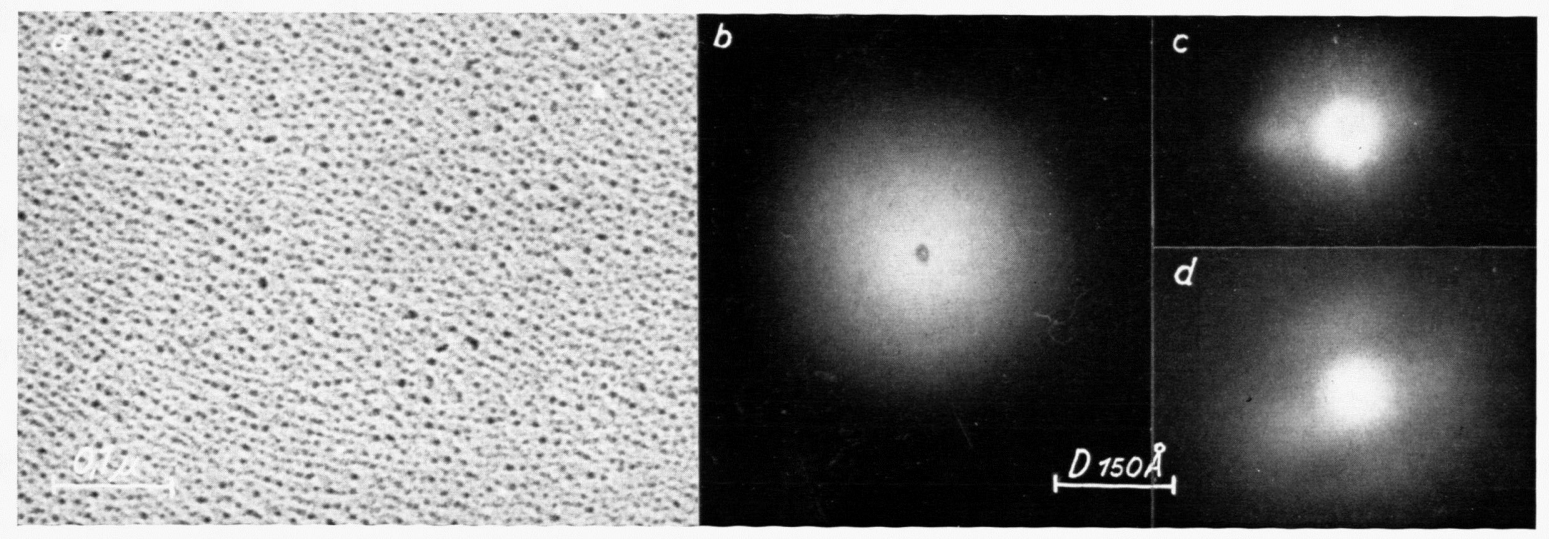

Abb. 8. Au auf NaCl-Spaltfläche bei $20^{\circ} \mathrm{C}$ senkrecht aufgedampft. a) EM-Bild (feinkörnige Schicht), Vergr. $120000: 1$, b-d) KW-Diagramme, $d D=1800$. 

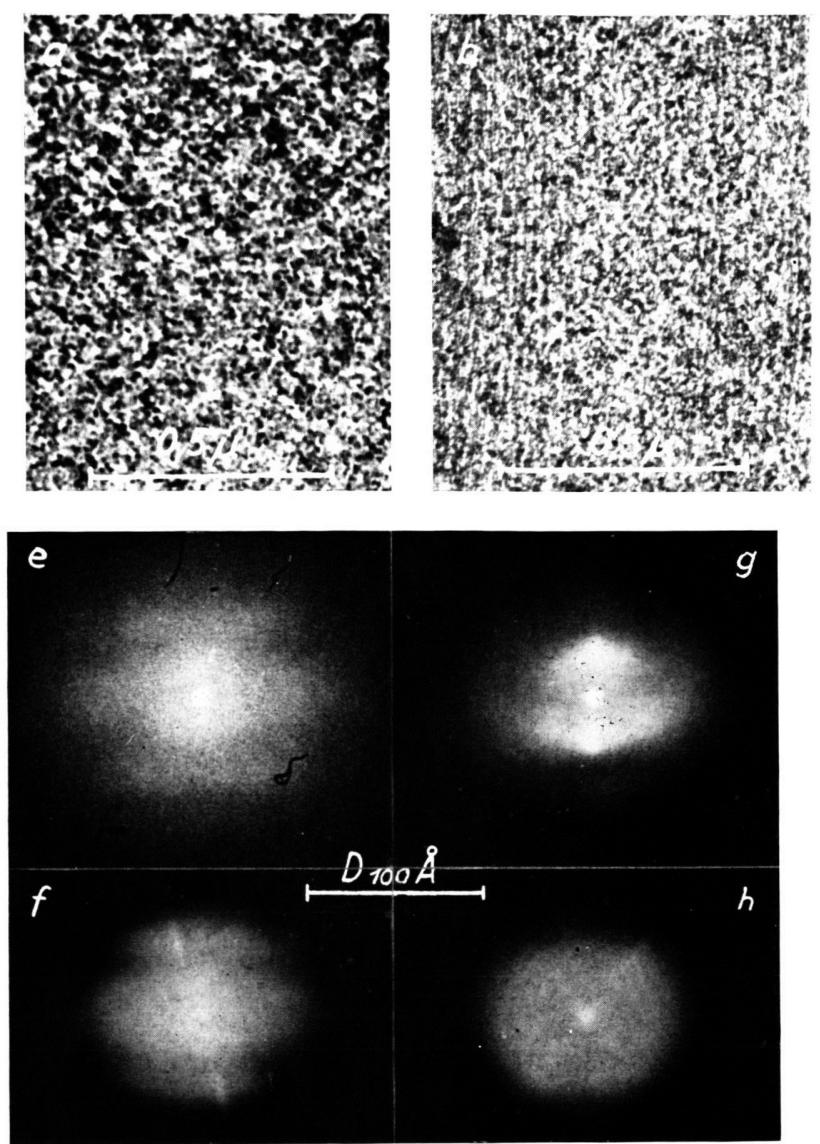
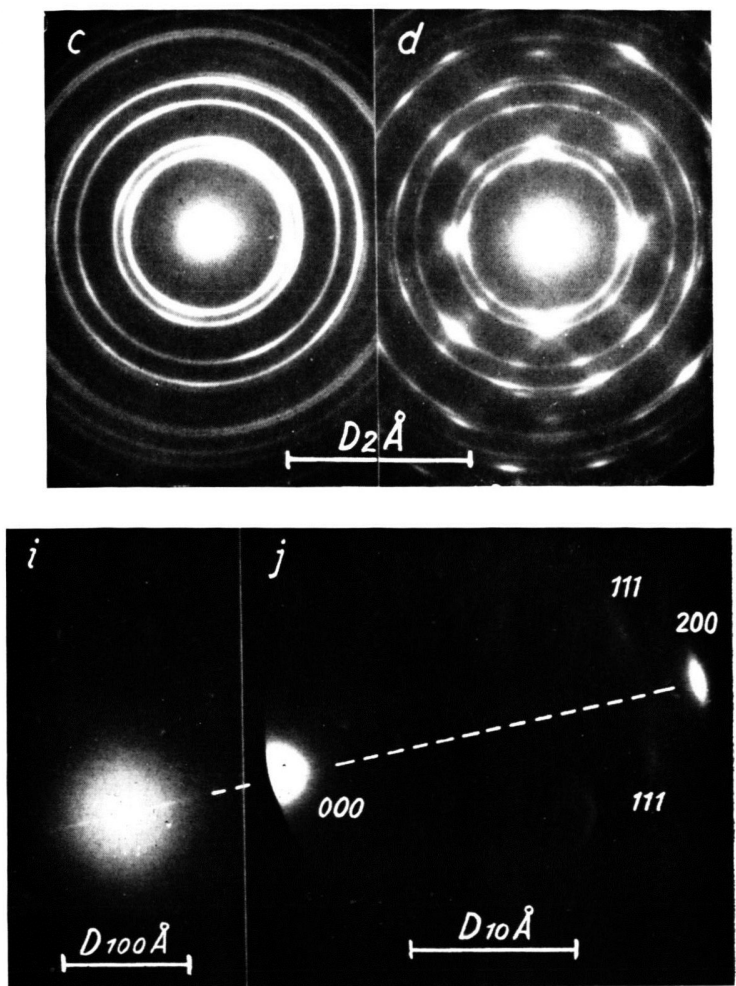

Abb. 9. Pd auf NaCl-Spaltfläche unter verschiedenen Bedingungen aufgedampft. a) und b) EM-Bilder, überwiegender Schichttyp (a), streifenförmige Stelle (b), Vergr. $50000: 1$; c) und d) Weitwinkeldiagramme von Schichten, die bei $20{ }^{\circ} \mathrm{C}$ senkrecht (c) und bei $300^{\circ} \mathrm{C}$ schäg (d) aufgedampft wurden. $d D=37$. e) $-\mathrm{j}$ ) KW-Diagramme von Schichten, die bei $20^{\circ} \mathrm{C}$ senkrecht (e und f, $d D=1800$ ), bei $200^{\circ} \mathrm{C}$ unter $45^{\circ}(\mathrm{g}, d D=1800$, gleiche Schicht wie Abb. $9 \mathrm{~b})$, bei $360{ }^{\circ} \mathrm{C}$ unter $45^{\circ}$ (h, $\left.d D=1800\right)$ und bei $250^{\circ} \mathrm{C}$ senkrecht (i, $d D=1300$ und $\mathrm{j}, d D=170$ ) aufgedampft wurden.

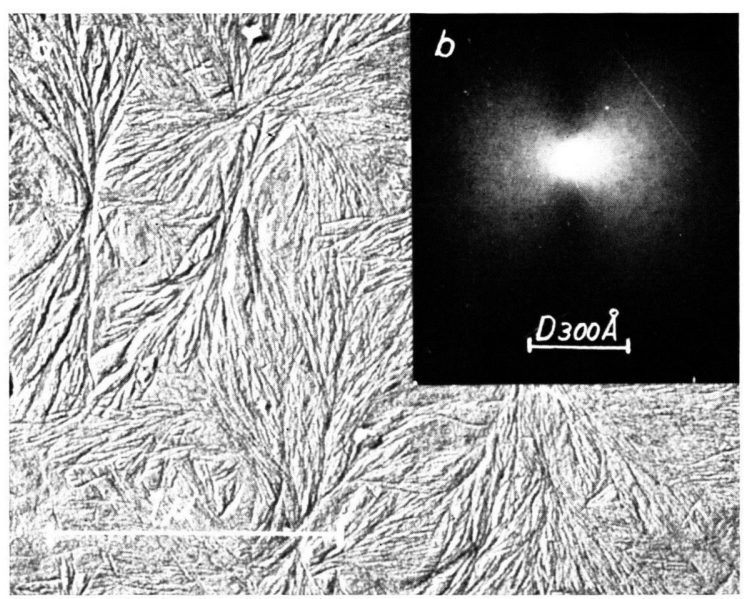

Abb. 10. Perlonfilm, schräg mit Pt bedampft. a) EM-Bild, Vergr. $25000: 1$; b) KW-Diagramm, $d D=3000$.

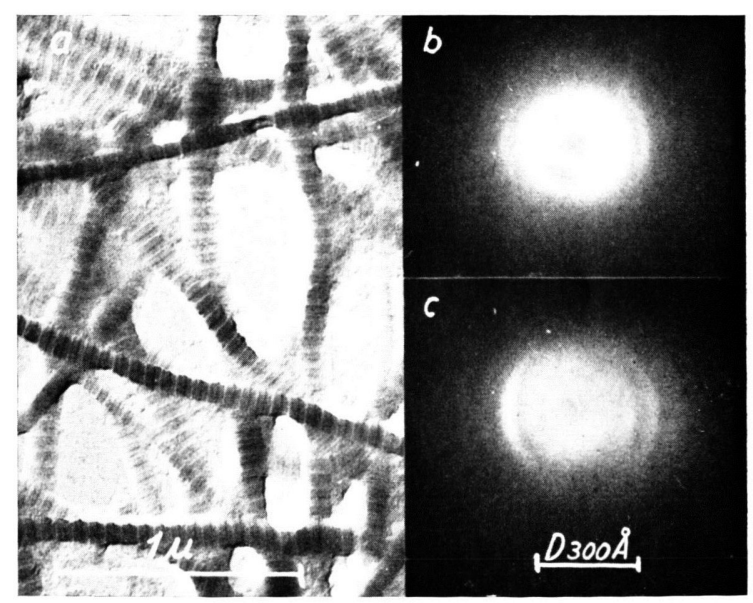

Abb.11. Kollagenfasern, schräg mit Pt bedampft. a) EM-Bild, Vergr. $25000: 1$; b) und c) KW-Diagramm, $d D=3000$. 
bar die etwa senkrecht zur Bedampfungsrichtung liegenden Strukturelemente bevorzugt hervorgehoben und führen zum Auftreten von Ringsegmenten. Diese lassen sich nach der BRAGGschen Gleichung mit guter Annäherung als 1., 2. und 3. Ordnung (Abb. 11 b) bzw. als 2., 3. und 4. Ordnung (Abb. $11 \mathrm{c}$ ) der bekannten Kollagenperiode von $640 \AA$ auffassen. Auf manchen Originalnegativen war noch die 5. Ordnung sichtbar, die auch in der Mikrophotometerkurve (Abb. 12) des Originalnegativs der Abb. $11 \mathrm{c}$ schwach hervortritt.

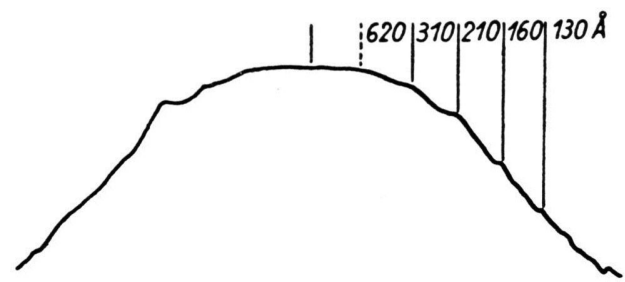

Abb. 12. Mikrophotometerkurve des Originalnegativs von Abb. $11 \mathrm{c}$.

\section{Ausblick}

Ob die hier beschriebene Methode mit Vorteil zur Strukturforschung einzusetzen ist, werden erst weitere Untersuchungen zeigen können. Für die Methode spricht vor allem die sehr viel größere Intensität der Elektronenstrahl-KW-Diagramme gegenüber den Röntgen-Strahl-KW-Diagrammen. Fast sämtliche der hier wiedergegebenen $\mathrm{KW}$-Beugungsdiagramme konnten visuell auf dem Leuchtschirm beobachtet werden; dementsprechend reichten für die Aufnahmen Belichtungszeiten von einigen Sekunden aus gegenüber vielen Stunden bei RöntGen-KW-Diagrammen. Darüber hinaus lassen sich in verschiedener Hinsicht Verbesserungsmöglichkeiten angeben.

10 F. Leonhard, Z. Naturforschg. 9 a, 1021 [1954].

11 H. Boersch, Optik 5, 436 [1949].
Einmal wird man versuchen, den störenden Streuuntergrund der Beugungsdiagramme zu vermindern, der besonders zum Primärfleck hin sehr stark ansteigt. Dies könnte mit einem rotierenden Sektor geschehen, wobei jedoch das KW-Diagramm wesentlich höher vergrößert werden müßte. Da der Untergrund, insbesondere in der Nähe des Primärflecks, großenteils aus unelastisch gestreuten Elektronen besteht (Leonhard ${ }^{10}$ ), könnte er durch GegenfeldFilterung erheblich verringert werden, was durch ein Filternetz (BoERSCH ${ }^{11}$ ) oder durch eine Filterlinse (Möllenstedt und RANG ${ }^{12}$ ) grundsätzlich zu erreichen ist.

Um ferner bei der Beobachtung isolierender, organischer Objekte einen so starken Eingriff wie die von uns erprobte Aufdampfung einer leitenden Schicht zu vermeiden, könnte man störende Aufladungen auf anderem Wege zu beseitigen suchen. Dazu ergeben sich als Möglichkeiten sowohl ein Entlader (KNöRzer ${ }^{13}$ ) als auch die Bestrahlung des Objekts mit langsamen Elektronen eines Geschwindigkeitsbereiches, in dem die Sekundärelektronenausbeute größer als 1 ist.

Schließlich ist es möglich, wie aus den Angaben in der Einleitung ${ }^{2,4}$ hervorgeht, die Auflösung noch um einen erheblichen Faktor zu steigern, um so eventuell auftretende schärfere Reflexe besser voneinander trennen zu können.

Für finanzielle Unterstützung der Untersuchungen sind wir dem Wirtschaftsministerium des Landes Baden-Württemberg und der Arbeitsgemeinschaft für Elektronenoptik zu Dank verpflichtet. Ferner danken wir Herrn Prof. Dr. G. Möllenstedt, Institut für experimentelle und angewandte Physik der Universität Tübingen, für die Aufnahmen der Mikrophotometerkurven.

12 G. Möllenstedt u. O. Rang, Z. angew. Phys. 3, 187 [1951].

13 G. KNörzer, Z. Naturforschg. 6 a, 511 [1951]. 